\title{
Young Adult Age-Period-Cohort Analysis of HIV and TB Mortality in South Africa: 1997-2015
}

\section{Tshifhiwa Nkwenika}

South Africa Medical Research Council

Samuel Manda ( $\nabla$ samuel.manda@mrc.ac.za)

South African Medical Research Council https://orcid.org/0000-0002-9672-3312

\section{Research}

Keywords: TB mortality, HIV mortality, APC analysis

Posted Date: August 24th, 2020

DOI: https://doi.org/10.21203/rs.3.rs-61574/v1

License: (c) (i) This work is licensed under a Creative Commons Attribution 4.0 International License. Read Full License 
Young Adult Age-Period-Cohort Analysis of HIV and TB Mortality in South Africa: 19972015

Tshifhiwa Nkwenika ${ }^{1}$, Samuel Manda ${ }^{1,2,3}$,

${ }^{1}$ Biostatistics Unit, South African Medical Research Council, Pretoria, South Africa ${ }^{2}$ School of Mathematics, Statistics and Computer Science, University of KwaZuluNatal, Pietermaritzburg, KwaZulu-Natal, South Africa

${ }^{3}$ Department of Statistics, University of Pretoria, Pretoria, South Africa

Correspondence to:

Samuel Manda

Email: samuel.manda@mrc.ac.za 


\section{Abstract}

Background: Young adult mortality is very significant in South Africa due to the influence of HIV/AIDS, Tuberculosis (TB), Injuries and Non-Communicable Diseases (NCDs). Previous analyses have mainly focused on assessing time effect of age and period separately. However, health outcomes often depend on three-time scales of age, period and cohort, which are linearly interlinked. Using Age-PeriodCohort (APC) models, this study estimated the time effects of age, period and cohort on HIV and TB mortality among young adults in South Africa.

Methods: HIV and TB mortality data and mid population estimates were obtained from Statistics South Africa for the period 1997 to 2015. Mortality data are based on deaths reported to the Department of Home Affairs where the underlying cause of death was HIV or TB based on the 10th revision of the International Statistical Classification of Diseases and Related Health Problems (ICD-10) definition. Observed HIV/AIDS deaths were adjusted for under-reporting, misclassification and systematic proportion from ill-defined natural deaths. Three-year age, period, and birth cohort intervals for 15-64 years, 1997-2015 and 1934-2000 respectively were used. The Age-Period-Cohort (APC) analysis using the Poisson distribution was used to compute the effects of age, period and cohort on mortality due to TB and HIV.

Results: A total of 5, 825,502 adult deaths were recorded from the period 1997 to 2015 of which, $910,731(15.6 \%)$ and 252,101 (4.3\%) were attributed to TB and HIV, respectively. For both observed mortality rate and estimated relative effects, concave down associations were found between TB, HIV mortality rates and period, age with 
peaks, at 36-38 and 30-32 years, respectively. A downward trend and inverted V-shape between TB and HIV mortality by birth cohort was found, respectively.

Conclusions: The study found an inverse U-shaped association between TB-related mortality and age, period, and general downward trend with birth cohort for deaths reported between 1997 and 2015. A concave down relationship between HIV-related mortality and age, period, and inverted V-shaped with birth cohort was found. Our findings have shed more light on HIV and TB mortality rates across different age groups, effect of changes in the overall TB and HIV management and care on the mortality rates and whether or not the mortality rates depended on the year an individual was born.

Keywords: TB mortality, HIV mortality, APC analysis 


\section{Background}

In South Africa, HIV/AIDS and TB are the leading infectious cause of death since 1997 and this remains unchanged ${ }^{1-3}$. The rates of mortality due to both HIV/AIDS and TB combined rapidly increased from 1997, reached a peak during the period 2006, and then started declining over time $^{1}$. This is observed in the age-specific, gender-specific, and cause-specific analysis, as exemplified by the annual mortality report given by the Statistics South Africa ${ }^{1}$, South African Medical Research Council (SAMRC) ${ }^{4}$ and the World Health Organization (WHO) ${ }^{5}$.

Mortality measures, such as age-specific, gender-specific death rates, longevity after birth, and the main causes of death, are critical for public health planning, program implementation, progress examination, making decisions, and asset allocation at both national and subnational level ${ }^{1,4,6,7}$. Information about young adult mortality serves an important purpose in South Africa in providing dependable population estimates and forecasts that aid effective planning in any sector, especially with the impact of emerging infectious diseases ${ }^{2,4,7}$. To yield mortality rates, population estimates are required. However, vital registration quality issues including the proportion of ill-defined causes that are high, misclassified HIV/AIDS deaths and poor allocation of underlying cause of death have been identified as limiting factors in South Africa ${ }^{2,4,8-11}$.

Over the decades, indirect statistical techniques such as Brass Growth Balance, Generalised Growth Balance, Bennett-Horiuchi, or Preston-Coale methods have been used to estimate the completeness of death registrations ${ }^{12-14}$. These techniques are adequately utilized by national statistical offices as they involve the use of census data and better knowledge of the distinct techniques. However, Adair and Lopez ${ }^{9}$ developed a practical approach to determine death registration completeness. The approach utilizes information often accessible at the sub-national and national levels. This approach of estimation has advantages over other statistical methods. These advantages include better accuracy, improved timeliness, application to subnational areas, improving mortality information at the country level by 
demographic characteristics, and greater simplicity of use ${ }^{10}$. Taking these advantages into account to adjust for under-reporting and misclassification of HIV/AIDS deaths, our study adopted the indirect descriptive statistics, an approach previously utilized by Groenewald ${ }^{10}$ and Fazito ${ }^{15}$.

Studies of long-term mortality patterns by demographic characteristics have proved helpful in understanding mortality rates. Further statistical analysis could provide better insights into the effects of demographic characteristics on mortality over time. In South Africa, STATSSA ${ }^{1}$, Dorrington et al. ${ }^{16}$, Groenewald et $\mathrm{al}^{8}$ have conducted and published studies which provide insight into mortality according to age, gender, race, as well as by other specific causes. including studies by.

Descriptive methods such as estimating the mortality rates by demographic characteristics and analytical methods including survival models, logistic regression, and Poisson regression for count mortality data have also been advocated. These models provide a formal method to independently evaluate a possible association between mortality rate and independent factors affecting mortality of an exposed population. The disadvantage of these techniques is that they cannot simultaneously distinguish relationships between confounders and mortality rates. The age-period-cohort analysis (APC analysis) can be used to assess the effects of age, period, and birth cohorts simultaneously on the variation of TB and HIV-related mortality rates. The application of APC analysis may be useful in jointly describing the environmental and historical factors which affect individuals differently over time ${ }^{17,18}$. Therefore, age, period to an event and birth cohort should be taken into consideration to provide robust interpretations of death rates over the hierarchy of respective subgroups. Currently, despite the fact that age-period-cohort (APC) analysis is more effective in analyzing trends as compared to conventional cross-sectional analysis ${ }^{19-20}$, there are no known studies in South Africa that have examined trends of TB and HIV mortality using this method. To understand HIV and TB-related mortality in SA over the past decade to inform our understanding of TB mortality and the development of strategies needed to end the 
TB epidemic by 2035 . Therefore, this study used APC analysis to simultaneously estimate age, period and birth cohort effects on TB- related and HIV-related mortality.

\section{Methods}

\section{Data sources and variables}

TB and HIV-related specific mortality and Mid-year population estimates used in this study were obtained from Statistics South Africa. Department of Home Affairs (DHA) register deaths using the death notification forms while Statistics South Africa processes the data and compiles mortality reports annually ${ }^{1}$. Young adult (15-64 years) HIV and TB mortality data was analyzed for the period from 1997 to 2015. The codes used for TB and HIV-related deaths were A15-A19, U51-U52, and B20-B24 of the International Classification of Diseases (ICD-10) ${ }^{21}$. Mid-population estimates at the country level as well as at the provincial level from 2002 till recently have been compiled by the Statistics South Africa. However, total populations for 1997 to 2001 are unavailable, we therefore used an exponential growth rate from census 1996 to estimate the mid-population; $P_{t+n}=P_{t} e^{n r}$, where $P_{t}$ is the total population at time $t, n$ is the length of time, and $r$ is the rate of population growth. Preliminary analyses involved calculating age-specific and cause-specific mortality rates.

\section{Statistical models and data analysis}

Age-Period-Cohort analysis was adopted to investigate age effects adjusting for period and birth cohorts; period effects adjusting for age and birth cohorts; and birth cohort effects adjusting for age and period on TB and HIV-related mortality. Suppose $i(1, \ldots, I)$ represents the age groups, where age group 1 incorporates 15-17 years old, age group 2 incorporates 18 20 years old, and so forth; $j(1, \ldots, J)$ represents 3 year period, with period 1 being 1997-1999, period 2 being 2000-2002, following the same pattern; and $k(1, \ldots, K)$ represents birth cohort. These comprise 17 age groups, 7 period and $\mathrm{K}=23$ birth cohort taking into consideration that age and period are divided into equal space intervals. To model deaths due to TB and HIV (let $\mathrm{z}=1$ and $\mathrm{z}=2$ be deaths due to TB and HIV respectively). Mortality rate for age ( $i$ ), period (j), and cohort $(k)$ are given by $\boldsymbol{R}_{i j k z}=\boldsymbol{O}_{i j k z} / \boldsymbol{N}_{\boldsymbol{i j k}}$. 
Where $O_{i j k z}$ denotes the observed deaths due to disease $z$, for age group $i$ at a given period $j$ and born in birth cohort k and $N_{i j k}$ represent the total population at risk. Supposing that age, period and birth cohort has an additive effect on the log rate, the natural log can be modeled as

$$
\operatorname{In}\left(R_{i j k z}\right)=\alpha_{i z}+\beta_{j z}+\gamma_{k z}+\varepsilon_{i j k z}
$$

Where age effect is represented by $\alpha_{i z}$, period effect is represented by $\beta_{j z}$, cohort effect is represented by $\gamma_{k z}$ and $\varepsilon_{i j k z}$ is the error term. This can be viewed as a Generalized Linear Model (GLM) with a log-link, and the model can be written as

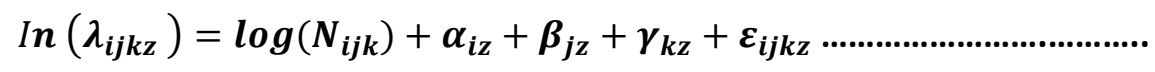

\section{(2)}

Where $\lambda_{i j k z}$ denotes the expected number of deaths due to $\mathrm{z}$ for age $(i)$ and period $(j)$ and born in the cohort $(k)$, that is assumed to follow a Poisson distribution. The APC model has a problem of being non-identifiable which arises because of the linear relationship between age, period, and birth cohort (Birth Cohort = Period - Age), Therefore, to solve the identification problem, our study adopted the intrinsic estimator (IE), which solves the problem of model identification and also provides unbiased and relatively efficient estimation results ${ }^{22,23}$. Adjusted data were used to estimate the effects of age, period and birth cohort on TB-related and HIV-related mortality.

\section{Results}

\section{Descriptive analysis}

The trends of age-specific (observed) TB and HIV-related mortality rates in South Africa from 1997 to 2015 are shown in Figure 1. A concave down association between TB mortality and 
period, with a peak during 2006 was observed while an upward trend was observed for HIVrelated mortality. Figure 2 and Figure 3 show the observed and revised age-specific HIVrelated death rates plotted for ages 15-64 years and by three age groups respectively, where an inverted U-shape was found.

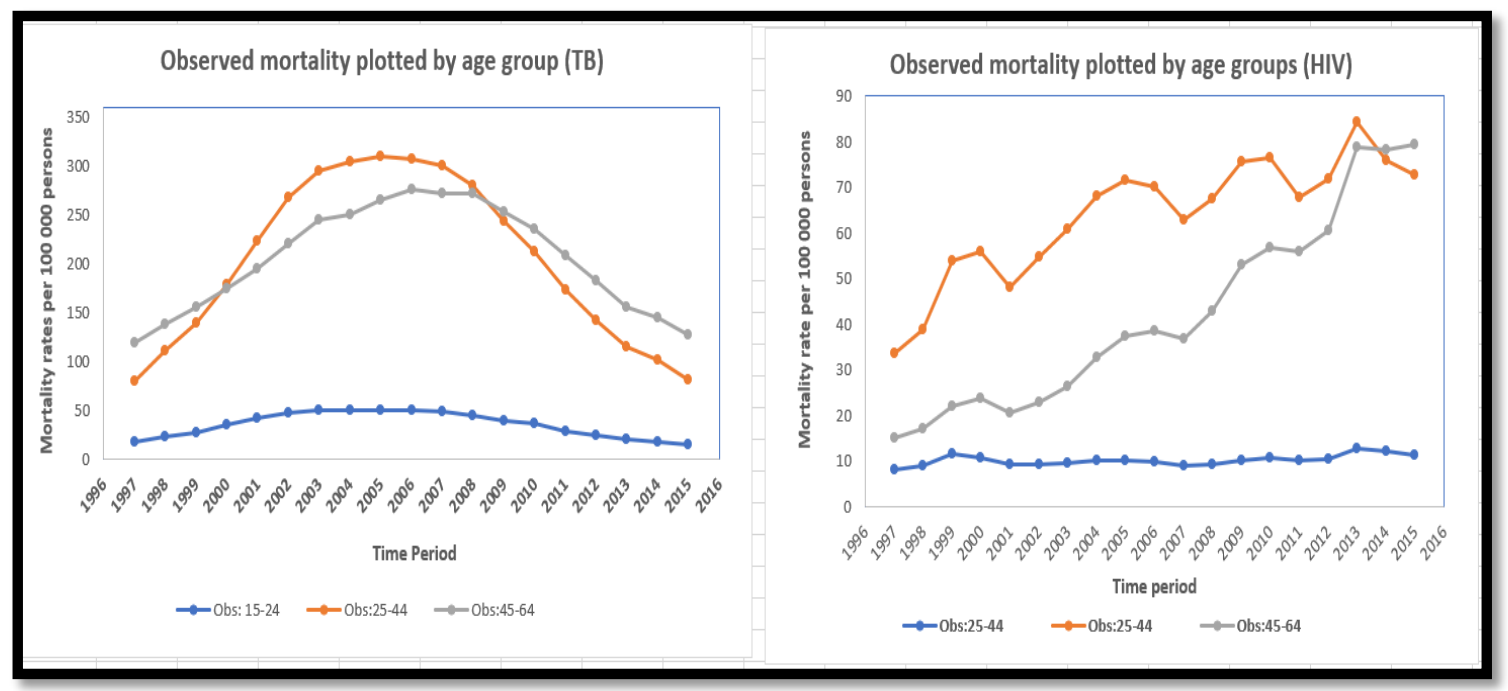

Figure 1: Observed TB-related and HIV-related mortality rates of the three age-groups

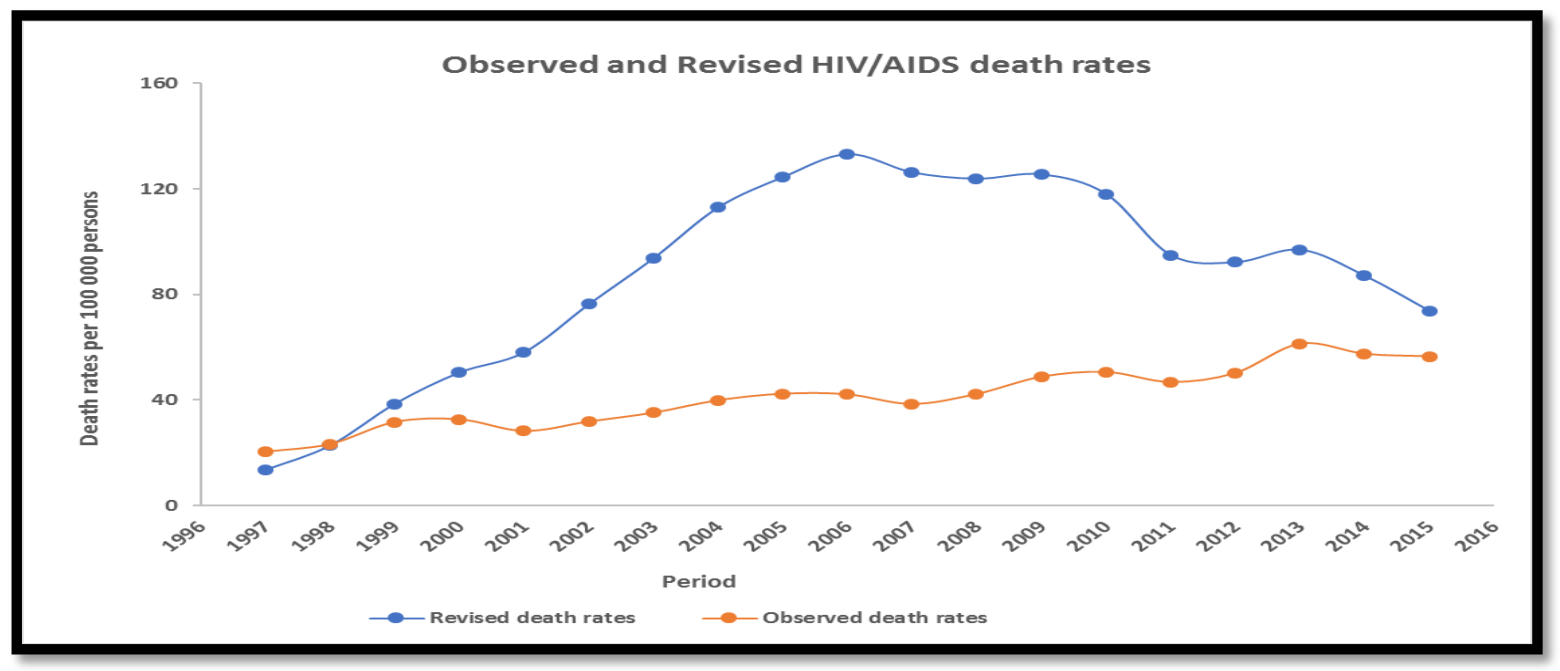

Figure 2: Observed and revised HIV-related mortality rates: 15-64 years 


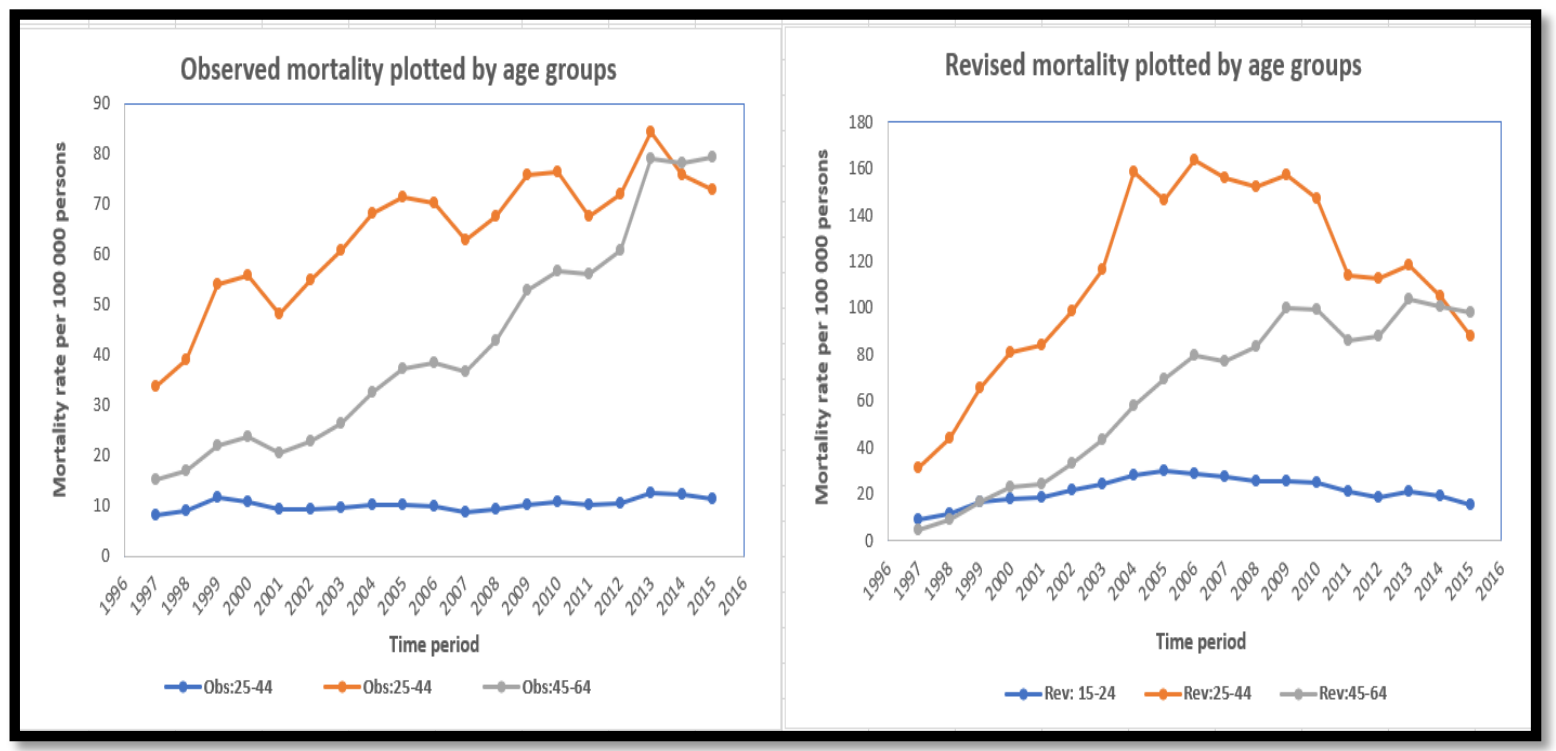

Figure 3: Observed and revised HIV-related mortality rates of the three age-groups

\section{Estimated Age, period, and birth cohort effects}

Table 1 and Table 2 show the estimated mortality relative risk for TB-related mortality and HIV-related mortality, as depicted in Figure 4 and Figure 5 respectively. Mortality relative risk and $95 \%$ confidence intervals are reported. Age, period, and cohort were statistically significantly associated with both TB and HIV-related mortality. Controlling for period and birth cohort effects, risks of TB-related mortality increased with individuals aging mutually, but it slows down after reaching the age between 33-35 years. The RRs of TB-related mortality also increased gradually by a period of observation, reached a peak by the year 2006-2008, and thereafter declined until 2015, holding age and birth cohort effects constant. An aggregated effect of exposure for different birth cohorts was estimated, a higher incidence of TB-related mortality was associated with an earlier cohort compared to later birth cohorts. The risk of HIV-related mortality increased with age peaking at 30-32 age group, controlling for period and birth cohort effects. Holding age and birth cohort effects fixed, a concave down association between period and HIV-related mortality was found. Birth cohort born before and after 1958-1960 and 1970-1972 were associated with lower HIV-related mortality compared to cohorts born between 1958 and1972.

Table 1: Age-period-cohort estimates for TB cause-specific mortality 


\begin{tabular}{|c|c|c|c|c|c|c|}
\hline \multirow{2}{*}{$\begin{array}{c}\text { TB Cause-Specific } \\
\text { Mortality }\end{array}$} & \multicolumn{3}{|c|}{ IE } & \multicolumn{3}{|c|}{ CGLIM } \\
\hline & \multirow[t]{2}{*}{$\mathbf{R R}$} & \multicolumn{2}{|c|}{$95 \% \mathrm{CI}$} & $\mathbf{R R}$ & \multicolumn{2}{|c|}{$95 \% \mathrm{CI}$} \\
\hline \multicolumn{6}{|l|}{ Age } & \\
\hline $15-17$ & 0.12 & 0.11 & 0.12 & 1.00 & - & - \\
\hline $18-20$ & 0.29 & 0.27 & 0.30 & 2.22 & 2.08 & 2.36 \\
\hline $21-23$ & 0.58 & 0.55 & 0.60 & 4.10 & 3.82 & 4.39 \\
\hline $24-26$ & 0.95 & 0.92 & 0.99 & 6.17 & 5.72 & 6.65 \\
\hline $27-29$ & 1.28 & 1.22 & 1.33 & 7.52 & 6.89 & 8.21 \\
\hline $30-32$ & 1.45 & 1.40 & 1.51 & 7.81 & 7.07 & 8.62 \\
\hline $33-35$ & 1.51 & 1.46 & 1.57 & 7.42 & 6.64 & 8.29 \\
\hline $36-38$ & 1.50 & 1.45 & 1.56 & 6.71 & 5.93 & 7.59 \\
\hline $39-41$ & 1.47 & 1.42 & 1.52 & 6.00 & 5.25 & 6.86 \\
\hline $42-44$ & 1.42 & 1.37 & 1.48 & 5.29 & 4.57 & 6.13 \\
\hline $45-47$ & 1.40 & 1.34 & 1.46 & 4.73 & 4.03 & 5.55 \\
\hline $48-50$ & 1.36 & 1.29 & 1.44 & 4.21 & 3.53 & 5.02 \\
\hline $51-53$ & 1.34 & 1.26 & 1.43 & 3.78 & 3.13 & 4.57 \\
\hline $54-56$ & 1.31 & 1.22 & 1.41 & 3.37 & 2.75 & 4.12 \\
\hline $57-59$ & 1.30 & 1.18 & 1.43 & 3.05 & 2.46 & 3.78 \\
\hline $60-62$ & 1.24 & 1.12 & 1.38 & 2.66 & 2.17 & 3.26 \\
\hline $63-64$ & 1.14 & 0.97 & 1.34 & 2.22 & 2.08 & 2.36 \\
\hline \multicolumn{7}{|l|}{ Period } \\
\hline $1997-1999$ & 0.60 & 0.56 & 0.64 & 1.00 & - & - \\
\hline $2000-2002$ & 1.10 & 1.08 & 1.12 & 2.01 & 1.87 & 2.16 \\
\hline $2003-2005$ & 1.50 & 1.46 & 1.53 & 3.00 & 2.77 & 3.25 \\
\hline $2006-2008$ & 1.58 & 1.55 & 1.61 & 3.47 & 3.20 & 3.77 \\
\hline $2009-2011$ & 1.25 & 1.23 & 1.27 & 3.01 & 2.76 & 3.29 \\
\hline $2012-2014$ & 0.81 & 0.79 & 0.84 & 2.14 & 1.93 & 2.38 \\
\hline 2015 & 0.63 & 0.61 & 0.66 & 1.84 & 1.63 & 2.06 \\
\hline \multicolumn{7}{|l|}{ Birth cohort } \\
\hline $1934-1936$ & 1.62 & 1.39 & 1.90 & 1.00 & - & - \\
\hline $1937-1939$ & 1.34 & 1.04 & 1.73 & 0.75 & 0.56 & 1.01 \\
\hline $1940-1942$ & 1.30 & 1.04 & 1.61 & 0.66 & 0.51 & 0.86 \\
\hline $1943-1945$ & 1.02 & 0.87 & 1.19 & 0.48 & 0.39 & 0.58 \\
\hline $1946-1948$ & 0.97 & 0.87 & 1.09 & 0.41 & 0.35 & 0.49 \\
\hline $1949-1951$ & 1.12 & 1.01 & 1.25 & 0.44 & 0.36 & 0.52 \\
\hline $1952-1954$ & 1.16 & 1.07 & 1.26 & 0.41 & 0.35 & 0.48 \\
\hline $1955-1957$ & 1.14 & 1.06 & 1.23 & 0.37 & 0.32 & 0.42 \\
\hline $1958-1960$ & 1.20 & 1.13 & 1.28 & 0.35 & 0.31 & 0.40 \\
\hline $1961-1963$ & 1.23 & 1.16 & 1.30 & 0.33 & 0.29 & 0.37 \\
\hline $1964-1966$ & 1.30 & 1.23 & 1.37 & 0.32 & 0.28 & 0.35 \\
\hline $1967-1969$ & 1.23 & 1.17 & 1.30 & 0.27 & 0.25 & 0.31 \\
\hline $1970-1972$ & 1.27 & 1.20 & 1.34 & 0.26 & 0.23 & 0.29 \\
\hline $1973-1975$ & 1.22 & 1.17 & 1.28 & 0.23 & 0.21 & 0.25 \\
\hline $1976-1978$ & 1.13 & 1.08 & 1.18 & 0.19 & 0.18 & 0.21 \\
\hline $1979-1981$ & 0.94 & 0.91 & 0.97 & 0.14 & 0.13 & 0.16 \\
\hline $1982-1984$ & 0.80 & 0.77 & 0.82 & 0.11 & 0.10 & 0.12 \\
\hline $1985-1987$ & 0.68 & 0.65 & 0.70 & 0.09 & 0.08 & 0.09 \\
\hline $1988-1990$ & 0.56 & 0.54 & 0.59 & 0.07 & 0.06 & 0.07 \\
\hline $1991-1993$ & 0.53 & 0.50 & 0.56 & 0.06 & 0.05 & 0.06 \\
\hline $1994-1996$ & 0.60 & 0.55 & 0.66 & 0.06 & 0.05 & 0.07 \\
\hline $1997-1999$ & 0.76 & 0.64 & 0.90 & 0.07 & 0.05 & 0.08 \\
\hline 2000 & 0.85 & 0.79 & 0.92 & 0.07 & 0.06 & 0.08 \\
\hline
\end{tabular}

Mortality relative risk (exp(b)) and 95\% confidence intervals are reported. Notes: ref group of each age, period, and birth cohort effect is the mean influence of all ages, periods, and birth cohorts combined.

Table 2: Age-period-cohort estimates for HIV cause-specific mortality 


\begin{tabular}{|c|c|c|c|c|c|c|}
\hline \multirow[b]{2}{*}{$\begin{array}{c}\text { HIV Cause-Spe cific } \\
\text { Mortality }\end{array}$} & \multicolumn{3}{|c|}{$\mathbf{I E}$} & \multicolumn{3}{|c|}{ CGLIM } \\
\hline & \multirow[t]{2}{*}{$\mathbf{R R}$} & \multicolumn{2}{|c|}{$95 \%$ CI } & $\mathbf{R R}$ & \multicolumn{2}{|c|}{$95 \%$ CI } \\
\hline \multicolumn{6}{|l|}{ Age } & \\
\hline $15-17$ & 0.47 & 0.42 & 0.54 & 1.00 & - & - \\
\hline $18-20$ & 0.52 & 0.50 & 0.55 & 1.09 & 0.95 & 1.25 \\
\hline $21-23$ & 0.70 & 0.67 & 0.74 & 1.44 & 1.24 & 1.68 \\
\hline $24-26$ & 1.31 & 1.27 & 1.36 & 2.65 & 2.26 & 3.11 \\
\hline $27-29$ & 1.07 & 1.04 & 1.11 & 2.14 & 1.81 & 2.52 \\
\hline $30-32$ & 1.66 & 1.61 & 1.71 & 3.25 & 2.73 & 3.87 \\
\hline $33-35$ & 1.61 & 1.56 & 1.66 & 3.10 & 2.59 & 3.72 \\
\hline $36-38$ & 1.53 & 1.48 & 1.58 & 2.91 & 2.41 & 3.51 \\
\hline $39-41$ & 1.44 & 1.40 & 1.49 & 2.70 & 2.22 & 3.28 \\
\hline $42-44$ & 1.40 & 1.33 & 1.48 & 2.59 & 2.10 & 3.18 \\
\hline $45-47$ & 0.81 & 0.74 & 0.88 & 1.47 & 1.17 & 1.84 \\
\hline $48-50$ & 0.78 & 0.74 & 0.83 & 1.40 & 1.12 & 1.75 \\
\hline $51-53$ & 0.78 & 0.75 & 0.82 & 1.38 & 1.10 & 1.73 \\
\hline $54-56$ & 0.82 & 0.77 & 0.87 & 1.43 & 1.12 & 1.81 \\
\hline $57-59$ & 0.89 & 0.84 & 0.95 & 1.52 & 1.19 & 1.95 \\
\hline $60-62$ & 1.05 & 0.98 & 1.13 & 1.77 & 1.38 & 2.27 \\
\hline $63-64$ & 1.29 & 1.13 & 1.47 & 2.14 & 1.81 & 2.52 \\
\hline \multicolumn{7}{|l|}{ Period } \\
\hline $1997-1999$ & 0.36 & 0.34 & 0.38 & 1.00 & - & - \\
\hline $2000-2002$ & 0.67 & 0.66 & 0.69 & 1.91 & 1.80 & 2.03 \\
\hline $2003-2005$ & 1.15 & 1.13 & 1.17 & 3.32 & 3.12 & 3.53 \\
\hline $2006-2008$ & 1.41 & 1.38 & 1.44 & 4.12 & 3.85 & 4.42 \\
\hline $2009-2011$ & 1.45 & 1.42 & 1.47 & 4.30 & 3.99 & 4.63 \\
\hline $2012-2014$ & 1.37 & 1.34 & 1.40 & 4.13 & 3.78 & 4.51 \\
\hline 2015 & 1.29 & 1.23 & 1.35 & 3.95 & 3.54 & 4.41 \\
\hline \multicolumn{7}{|l|}{ Birth cohort } \\
\hline $1934-1936$ & 0.39 & 0.35 & 0.45 & 1.00 & - & - \\
\hline $1937-1939$ & 0.32 & 0.26 & 0.40 & 0.81 & 0.64 & 1.03 \\
\hline $1940-1942$ & 0.61 & 0.43 & 0.87 & 1.51 & 1.01 & 2.25 \\
\hline $1943-1945$ & 0.89 & 0.74 & 1.08 & 2.17 & 1.73 & 2.72 \\
\hline $1946-1948$ & 1.09 & 0.98 & 1.20 & 2.60 & 2.31 & 2.92 \\
\hline $1949-1951$ & 1.30 & 1.21 & 1.40 & 3.06 & 2.73 & 3.43 \\
\hline $1952-1954$ & 1.48 & 1.37 & 1.60 & 3.43 & 3.07 & 3.84 \\
\hline $1955-1957$ & 1.61 & 1.51 & 1.72 & 3.68 & 3.35 & 4.03 \\
\hline $1958-1960$ & 1.90 & 1.75 & 2.05 & 4.26 & 3.88 & 4.67 \\
\hline $1961-1963$ & 2.08 & 1.94 & 2.22 & 4.60 & 4.27 & 4.95 \\
\hline $1964-1966$ & 2.22 & 2.10 & 2.34 & 4.83 & 4.56 & 5.12 \\
\hline $1967-1969$ & 2.09 & 1.98 & 2.22 & 4.49 & 4.24 & 4.75 \\
\hline $1970-1972$ & 1.93 & 1.83 & 2.03 & 4.07 & 3.85 & 4.30 \\
\hline $1973-1975$ & 1.74 & 1.67 & 1.82 & 3.63 & 3.45 & 3.82 \\
\hline $1976-1978$ & 1.58 & 1.51 & 1.65 & 3.24 & 3.05 & 3.44 \\
\hline $1979-1981$ & 1.29 & 1.24 & 1.35 & 2.61 & 2.45 & 2.79 \\
\hline $1982-1984$ & 1.05 & 1.00 & 1.11 & 2.09 & 1.93 & 2.27 \\
\hline $1985-1987$ & 0.85 & 0.81 & 0.88 & 1.66 & 1.52 & 1.81 \\
\hline $1988-1990$ & 0.70 & 0.67 & 0.72 & 1.34 & 1.21 & 1.49 \\
\hline $1991-1993$ & 0.61 & 0.57 & 0.65 & 1.16 & 1.01 & 1.32 \\
\hline $1994-1996$ & 0.54 & 0.50 & 0.58 & 1.01 & 0.86 & 1.17 \\
\hline $1997-1999$ & 0.44 & 0.40 & 0.48 & 0.80 & 0.67 & 0.96 \\
\hline 2000 & 0.38 & 0.34 & 0.43 & 0.69 & 0.56 & 0.86 \\
\hline
\end{tabular}

Mortality relative risk (exp(b)) and 95\% confidence intervals are reported. Notes: ref group of each age, period, and birth cohort effect is the mean influence of all ages, periods, and birth cohorts combined. 


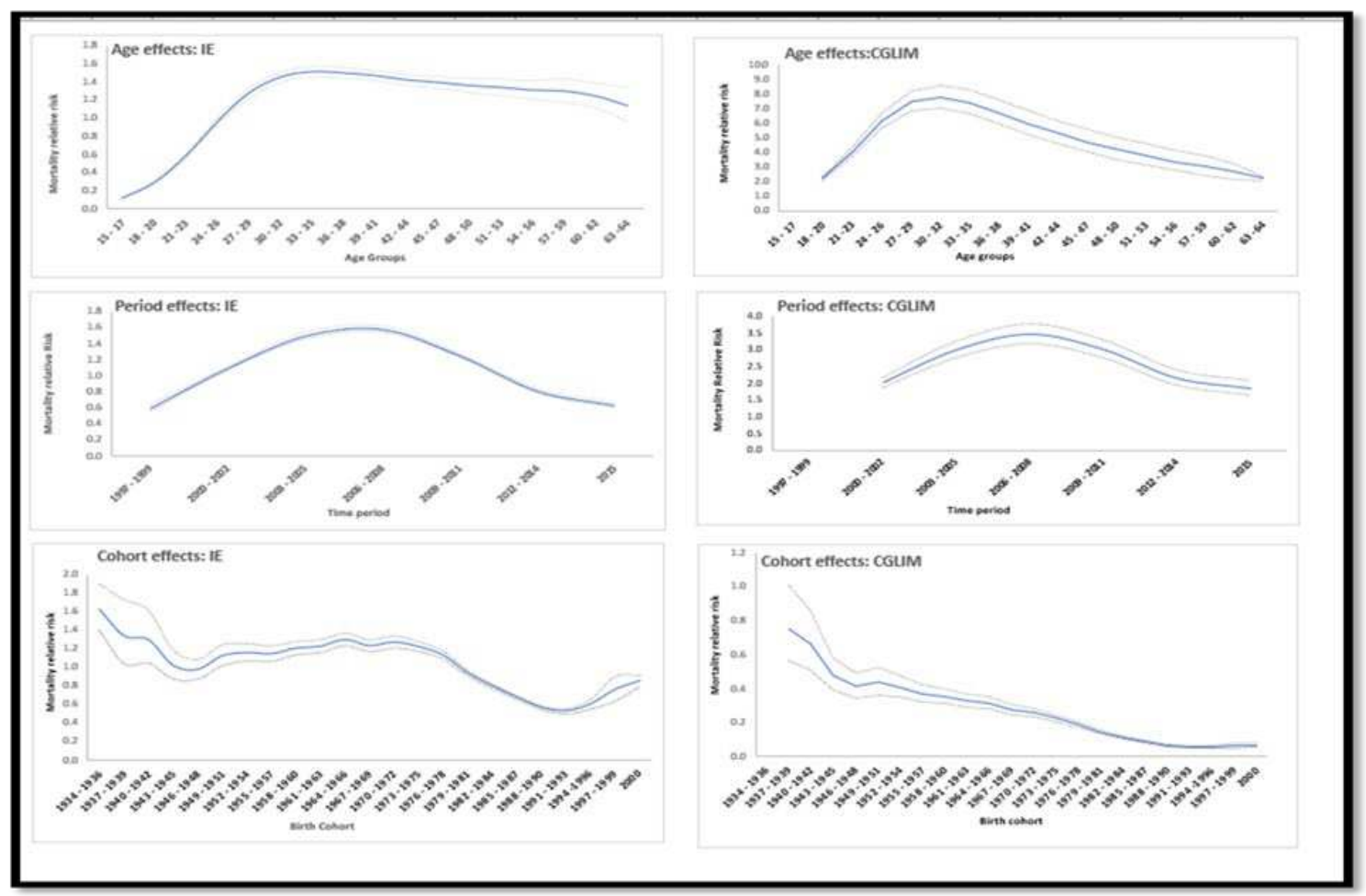

Figure 4: Age, period and cohort effects of TB mortality. Notes: dotted lines present $95 \%$ confidence intervals.

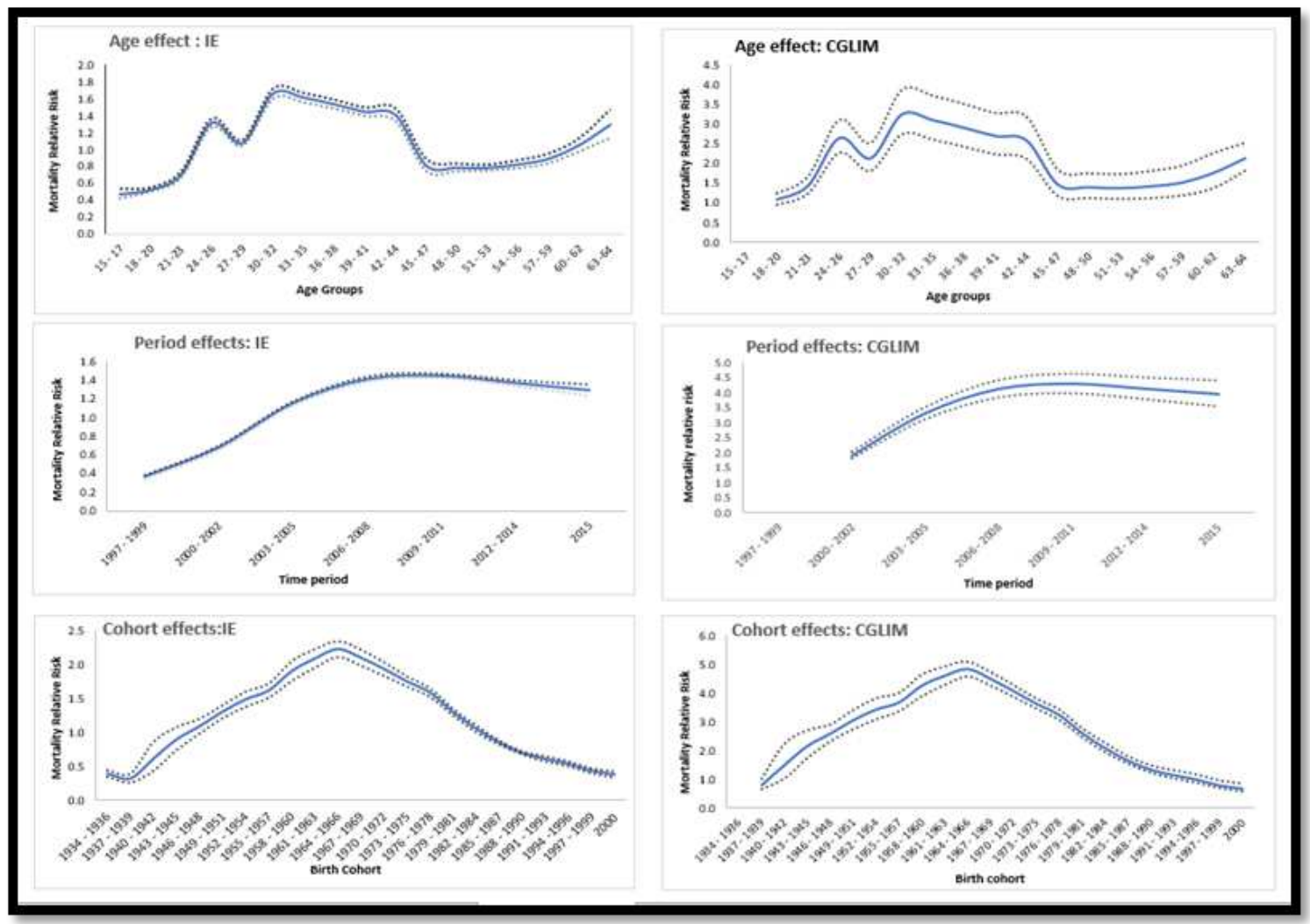

Figure 5: Age, period and cohort effects of HIV mortality. Notes: dotted lines present $95 \%$ confidence intervals. 


\section{Discussion}

This study was set out to simultaneously estimate the effects of age, period and birth cohort on mortality rates due to either TB or HIV among individuals between 15 and 64 years of age in South Africa. The Age-Period-Cohort (APC) framework analyzed through a Poisson regression model on the death counts with population exposed as offset was utilized. This model allowed us to assess the association between the mortality of either TB or HIV and attained age, calendar period, and birth cohort (year of birth). Official mortality data and mid population between 1997 and 2015, with three-year age and period intervals were used. The number of HIV/AIDS deaths were adjusted for misclassification and under-reporting.

We found a concave down association between age and TB mortality, with a peak at 33-35 years. There was a concave down relationship between TB cause-specific mortality in the studies of mortality data period between 1997 and 2015. There was a downward trend between TB mortality and the effect of birth cohort from 1934 to 2000. There was an inverse flatter U-shaped association between age and HIV mortality and it was more pronounced at 30-32 years. The estimated relative risks showed an inverse U-shaped relationship between HIV mortality and the effect of the period from 1997 to 2015. An inverted V-shape relationship between birth cohort and HIV-related mortality was observed.

Estimated relative risk of TB and HIV mortality rates using the APC analysis found similar results as official statistics, global TB and HIV report, and other similar research findings in South Africa have shown about age effects ${ }^{1,2,6,24,25}$. However, our estimated RRs for HIVrelated mortality differed from the official statistics of South Africa in terms of period effects. Our study showed that the age group 33-35 was more at risk of TB mortality than all other age groups, which correspond to Kootbodien et.al ${ }^{25}$ findings. We found a decline in TB death rates in South Africa from 2007 to 2015, which is in line with the $68.8 \%$ to $77.2 \%$ global reduction in TB mortality from 2007 to 2014 respectively. A similar trend for TB deaths 
overtime was reported by the official statistics of South Africa ${ }^{1}$ which was also confirmed by other researchers ${ }^{2,6,26}$. Meanwhile, there are possible explanations for the decreasing TB death rates over time among young adults in South Africa. Such a rapid decrease may be due to an increase in treatment success rate $(83 \%)$ achieved in the year 2016 and reported by the National TB statistics for South Africa. The decline in the change rate over time represents improvements in the initiatives taken to mitigate the burden of TB mortality $5,27,28$. The incidence of TB mortality was higher in the earlier cohorts compared to later cohorts. Such could be a result of the improvement in the living standards and health circumstances of young adult cohorts and their subjection at early ages under these favorable incidences ${ }^{29}$. This may also be associated with better health equipment and medical administration during the $21^{\text {st }}$ century. This study showed that later birth cohorts had a lesser risk of TB mortality compared to earlier birth cohorts. Therefore, we recommend that vaccination, screening, and treatment coverage be increased among earlier birth cohorts.

This study has shown a higher risk of HIV mortality among the age groups 30-32, 33-35, and 36-38 years compared to all other age groups. A study by Haal et al ${ }^{30}$ supports these current findings. They also found the age group 19-39 to be more at risk of HIV-related mortality in South Africa. Pillay-van Wyk et.al ${ }^{2}$ and Haal et.al. ${ }^{30}$ reported an inverse U-shaped relationship between HIV/AIDS deaths and period. Factors such as the introduction of the antiretroviral (ARV) treatment programme at the public health sectors ${ }^{29}$ and an improvement in the completeness of vital registration with less misclassification over time ${ }^{1}$ may have accounted for a decrease in HIV/AIDS mortality. This study indicated a higher HIV mortality risk for birth cohort 1946-1949 to 1982-1984 with even greater risk for birth cohort 19641966. Our findings agree with the world mortality report by the UN, where it is indicated that mortality risks due to HIV/AIDS compared to all causes of mortality rates were higher among 1950 to 1955 birth cohort. Butler et.al. ${ }^{31}$ confirmed a higher HIV/AIDS mortality among the older birth cohort of South Africa. Factors like time to exposure, weak immune system, and lack of education may have contributed to a greater risk of HIV-related mortality among older birth cohort ${ }^{31}$. 
The findings and subsequent discussions are subject to the limitations of our study. Officially published mortality data based on the underlying cause of death were used. Furthermore, mortality data are under-reported, misclassified, and incomplete ${ }^{4}$, which could have affected and confounded the observed rates and their interpretation. Given these limitations, mortality estimates should be interpreted with caution.

The study found an inverse U-shaped association between TB-related mortality and age, period, and a general downward trend with birth cohort for deaths reported between 1997 and 2015. A concave downward relationship between HIV-related mortality and age, period, and inverted V-shaped with birth cohort was found. The association between HIV-related mortality and period differs from the officially reported trend with adjustment, which shows an upward progression. Our findings are based on a slightly advanced APC statistical model . Using APC analysis, we found secular trends in TB and HIV-related mortality rates which could contribute to certain guides in long-term planning, monitoring, and evaluation in health establishments.

\section{Conclusions}

Even though both HIV and TB mortality rates have been falling in South Africa globally, they remain among the top five leading causes of natural deaths among the country's young adults. South Africans. Our findings based on Age-Period-Cohort analyses have simultaneously identified the mortality rates across different age groups, effect of period as it relates to changes in the overall TB and HIV health systems, management and care and cohort effects to capture effect of prevailing economic and health contextual determinants at the year of birth on risk of HIV and TB mortality. As these two diseases have a high co-morbidity both at individual and 
ecological levels, our findings here would help the South African National Department of Health with valuable evidence on which to monitor and measure progress in reducing tuberculosis (TB) mortality rates in line with the World Health Organization's End TB Strategy and the Sustainable Development Goal (SDG).

\section{List of Abbreviations}

AIC - Akaike Information Criterion; AIDS - Acquired Immune Deficiency Syndrome; APC - Age Period Cohort Analysis; ARV - Antiretroviral BIC - Bayesian information criterion; CGLM Constrained Generalized Linear Models; DHA - Department of Home Affairs; DOH Department of Health; HIV- Human Immunodeficiency Virus; ICD- International Classification of Diseases; IE - Intrinsic Estimator; PCA - Principal Component Analysis; SAMRC - South African Medical Research Council; STATSSA- Statistics South Africa; TB - Tuberculosis; UN United Nations; WHO - World Health Organization

\section{Ethical approval and consent to participate}

Data used are publicly accessible from http://www.datafirst.uct.ac.za/dataportal/ include no personal identifiers. Thus, ethical issues and consent required were dealt with by the respective study team.

\section{Consent for publication}

Not applicable.

\section{Availability of data and materials}

Anonymized versions of the datasets analyzed in this study are available at the Data first, (http://www.datafirst.uct.ac.za/dataprotal/index.php/catalog/).

\section{Competing interests}

The authors declare no conflict of interest

\section{Funding}

The study was funded by the South African Medical Research Council.

\section{Author's contributions}

SM conceptualized and designed the study and statistical analysis plans. TN conducted the literature review, performed data management, analysis and wrote the initial draft. Both authors interpreted the results and contributed to subsequent revisions.

\section{Acknowledgment}

South African Medical Council for funding this study. We thank Statistics South Africa for Compiling and publishing data that we used.

\section{Author's information}

${ }^{1}$ Biostatistics Unit, South African Medical Research Council, Pretoria, South Africa, Pretoria. ${ }^{2}$ School of Mathematics, Statistics and Computer Science, University of KwaZulu-Natal, Pietermaritzburg, KwaZulu-Natal, South Africa.

\section{References}


1. Statitsics South Africa. Mortality and causes of death in South Africa, 2016: Findings from death notification. Stat release. 2016;(November 2016):1-127. doi: Statistical release P0309.3

2. Pillay-van Wyk V, Msemburi W, Laubscher R, et al. Mortality trends and differentials in South Africa from 1997 to 2012: second National Burden of Disease Study. Lancet Glob Heal. 2016;4(9):e642-e653. doi:10.1016/S2214-109X(16)30113-9

3. Adeiza MA, Abba AA, Okpapi JU. HIV-Associated Tuberculosis : A sub-Saharan African Perspective. 2014;1(1).

4. Bradshaw D, Pillay-Van Wyk V, Laubscher R, et al. Cause of death statistics for South Africa: Challenges and possibilities for improvement. 2010;(November).

5. $\quad$ United Nations. World Mortality Report 2015 - Highlights.; 2015.

6. $\quad$ Phillips JA. Global Tuberculosis. Vol 63.; 2015. doi:10.1177/2165079915607875

7. Statistics South Africa. Statistical release Mortality and causes of death in South Africa, 2014: Africa Pretoria Stat South Africa. 2014;(November). doi:Statistical release P0309.3

8. Groenewald P, Neethling I, Evans J, et al. Mortality trends in the city of Cape Town between 2001 and 2013: Reducing inequities in health. South African Med J. 2017;107(12):1091-1098. doi:10.7196/SAMJ.2017.v107i12.12458

9. Adair T, Lopez AD. Estimating the completeness of death registration: An empirical method. PLoS One. 2018;13(5):1-19. doi:10.1371/journal.pone.0197047

10. Groenewald P, Bradshaw D, Dorrington R, Bourne D, Laubscher R, Nannan N. Identifying deaths from AIDS in South Africa: An update [4]. Aids. 2005;19(7):744-745.

doi:10.1097/01.aids.0000166105.74756.62

11. Birnbaum JK, Murray CJL, Lozano R. Présentation du classement incorrect des décès liés au VIH/SIDA en Afrique du Sud. Bull World Health Organ. 2011;89(4):278-285. doi:10.2471/BLT.11.086280

12. Dorrington R, Bradshaw D, Wegner T. Estimates of the Level and Shape of Mortality Rates in South Africa Around 1985 and 1990 Derived by Applying Indirect Demographic Techniques to Reported Deaths. 1999;(April):1-101.

13. Bennett NG, Horiuchi S. Estimating the Completeness of Death Registration in a Closed Population. Popul Index. 1981;47(2):207. doi:10.2307/2736447

14. da Silva Escada RO, Velasque L, Ribeiro SR, et al. Mortality in patients with HIV-1 and tuberculosis co-infection in Rio de Janeiro, Brazil - associated factors and causes of death. BMC Infect Dis. 2017;17(1):1-10. doi:10.1186/s12879-017-2473-y

15. Fazito $E$, Cuchi $P$, Fat $D M$, et al. Identifying and quantifying misclassified and underreported AIDS deaths in Brazil: a retrospective analysis from 1985 to 2009. Sex Transm Infect. 2012;88 Suppl 2:86-94. doi:10.1136/sextrans-2012-050632

16. Bradshaw D, Dorrington R. Rapid Mortality Surveillance Report 2011.; 2012.

17. Schulhofer-wohl S, Land KC. Yang_etal_AJS.pdf. 2008;113(6):1697-1736.

18. Keyes KM, Utz RL of U, Robinson W, Li G. What is a cohort effect? Soc Sci Med. 2012;70(7):1100-1108. doi:10.1016/j.socscimed.2009.12.018. What

19. Chang YH, Li CY, Tung TH, Tsai JJ, Lu TH. Age-period-cohort analysis of mortality from epilepsy in Taiwan, 1971-2005. Seizure. 2011;20(3):240-243. doi:10.1016/j.seizure.2010.12.001

20. Gao X, Wang Z, Kong C, Yang F, Wang Y, Tan X. Trends of esophageal cancer mortality in rural China from 1989 to 2013: An age-period-cohort analysis. Int J Environ Res Public Health. 2017;14(3):1-10. doi:10.3390/ijerph14030218

21. World Health Organization. International statistical classification of diseases and related health problems, 10th revision (ICD-10). World Heal Organ. 2016;1(Chapter V):332-345. http://www.who.int/classifications/icd/icdonlineversions/en/.

22. Yang Y, Schulhofer-Wohl S, Fu WJ, Land KC. The intrinsic estimator for age-period-cohort analysis: What it is and how to use it. Am J Sociol. 2008;113(6):1697-1736. doi:10.1086/587154

23. Luo L, Hodges J. The cross-classified age-period-cohort model as a constrained estimator. Under Rev. 2013;(November). http://paa2013.princeton.edu/papers/132093. 
24. Msemburi W, Pillay, Wyk V, et al. Second National Burden of Disease Study for South Africa: Cause-of Death Profile for South Africa, 1997-2012.; 2016.

http://www.mrc.ac.za/sites/default/files/files/2016-12-08/SouthAfrica2012.pdf.

25. Kootbodien T, Wilson $\mathrm{K}$, Tlotleng $\mathrm{N}$, et al. Tuberculosis mortality by occupation in South Africa, 2011-2015. Int J Environ Res Public Health. 2018;15(12).

doi:10.3390/ijerph15122756

26. Day C, Gray A, Budgell E. Health and related indicators. South African Heal Rev. 2011;2011(1):119-248.

27. Dorrington R, Bradshaw D, Laubscher R. Rapid Mortality Surveillance Report 2012.; 2014. doi:ISBN: 978-1-920618-00-1

28. Karim SSA, Churchyard GJ, Karim A, Lawn SD. Escalate the Public Health Response. 2010;374(9693):921-933. doi:10.1016/S0140-6736(09)60916-8.HIV

29. Onyango DO, Yuen CM, Cain KP, Ngari F, Masini EO, Borgdorff MW. Reduction of HIVassociated excess mortality by antiretroviral treatment among tuberculosis patients in Kenya. PLoS One. 2017;12(11):1-13. doi:10.1371/journal.pone.0188235

30. Haal K, Smith A, van Doorslaer E. The rise and fall of mortality inequality in South Africa in the HIV era. SSM - Popul Heal. 2018;5(February):239-248.

doi:10.1016/j.ssmph.2018.06.007

31. Butler I, MacLeod W, Majuba PP, Tipping B. Human immunodeficiency virus infection and older adults: A retrospective single-site cohort study from Johannesburg, South Africa. South Afr J HIV Med. 2018;19(1):1-8. doi:10.4102/sajhivmed.v19i1.838

\section{Appendix I}

\section{Methods for adjustments of HIV/AIDS mortality}

For this study, 'misclassified' deaths are defined as deaths that were due to AIDS but not reported as such in the death certificates, while 'Under-reported' deaths refers to deaths which were not declared or registered in the civil registries. According to the ICD-10 underlying cause of death selection rules, a death may be considered as HIV/AIDS if there is reference to HIV/AIDS in any line of the death certificate, as well as mention of a condition that is considered a direct consequence of HIV/AIDS. The conditions that are assumed to be direct consequences of HIV/AIDS are Kaposi's sarcoma, Burkitt's tumour, and any other malignant neoplasm of lymphoid, haematopoietic and related tissue, classifiable to C46 or C81-96; and any infectious diseases classifiable to A00-B19, B25-B49, B58-B64, B99 or J12-J18 [26]. To adjust for misclassification, the potential 28 causes of death were investigated using age-specific rates trends plotted over time. AIDS-indicators following the same distinct age pattern typical of HIV/AIDS age-specific death rates trends plotted over time were selected for estimating excess mortality. Statistics South Africa [27] indicated that $96 \%$ of completeness of death registration was achieved for the 2015 period. This is significant given that we utilized the 2015 death rate to calculate expected mortality (expected mortality was subtracted from observed mortality) and excess mortality from the causes which were identified as AIDS-indicators. Death records with no information on age were redistributed proportionally to all other age groups for the same year of 
death while deaths due to ill-defined causes were redistributed across all other natural causes of death. Expected numbers of AIDS deaths that were coded as ill-defined causes were estimated using the proportion of AIDS deaths among all-natural causes of deaths. To adjust for under-reporting, the observed number of AIDS deaths were further adjusted for incompleteness. To do that, the ratio of overall observed death rate from our working data and overall estimated death rate from WHO was subtracted from 1. Furthermore, excess and expected mortality due to ill-defined causes were scaledup by the proportion of incompleteness. 
Figures

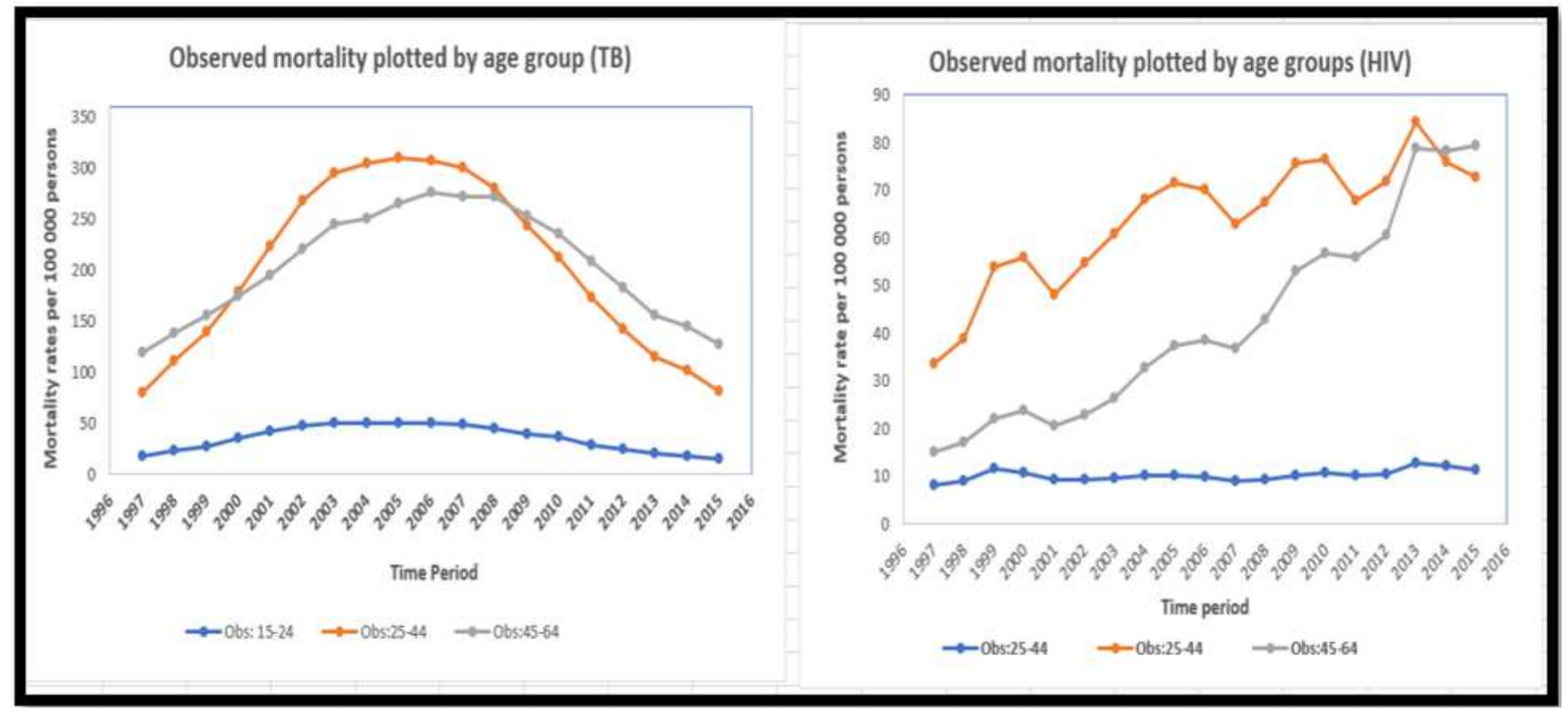

Figure 1

Observed TB-related and HIV-related mortality rates of the three age-groups

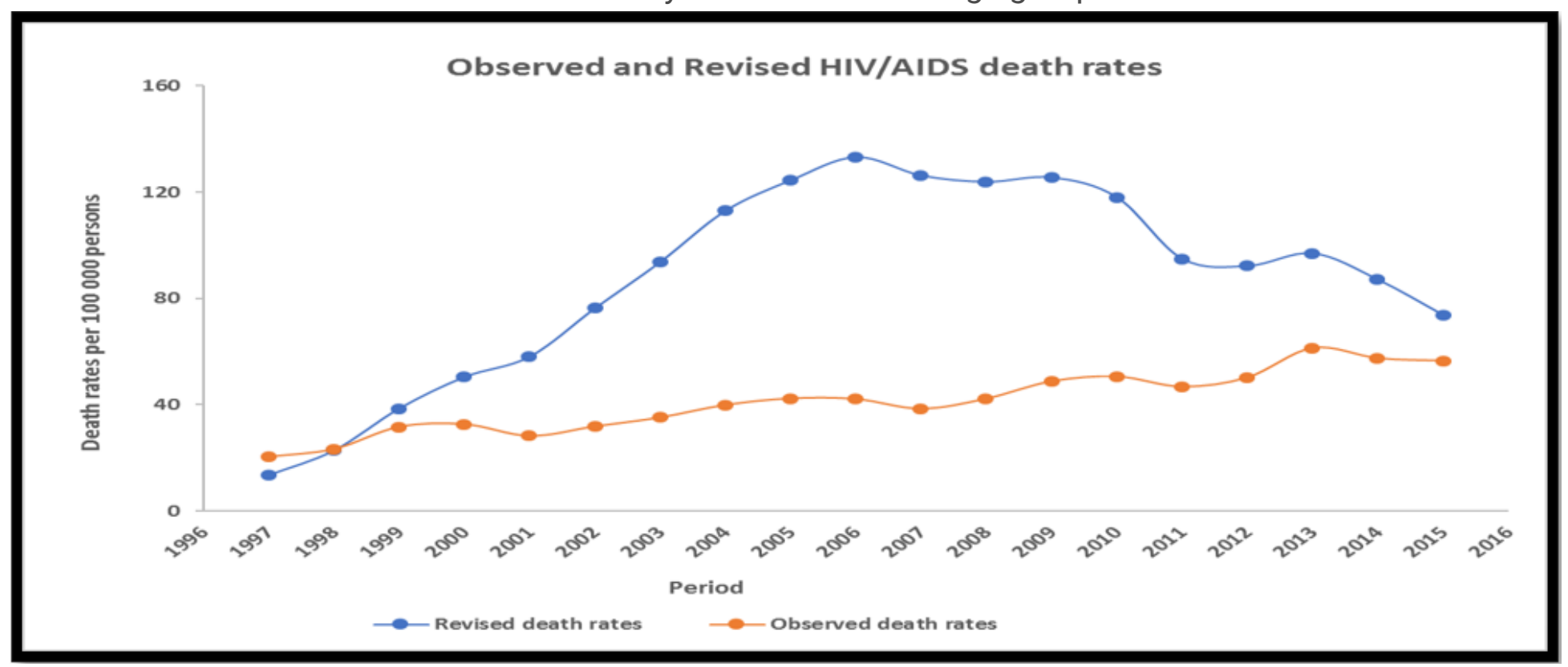

Figure 2

Observed and revised HIV-related mortality rates: 15-64 years 


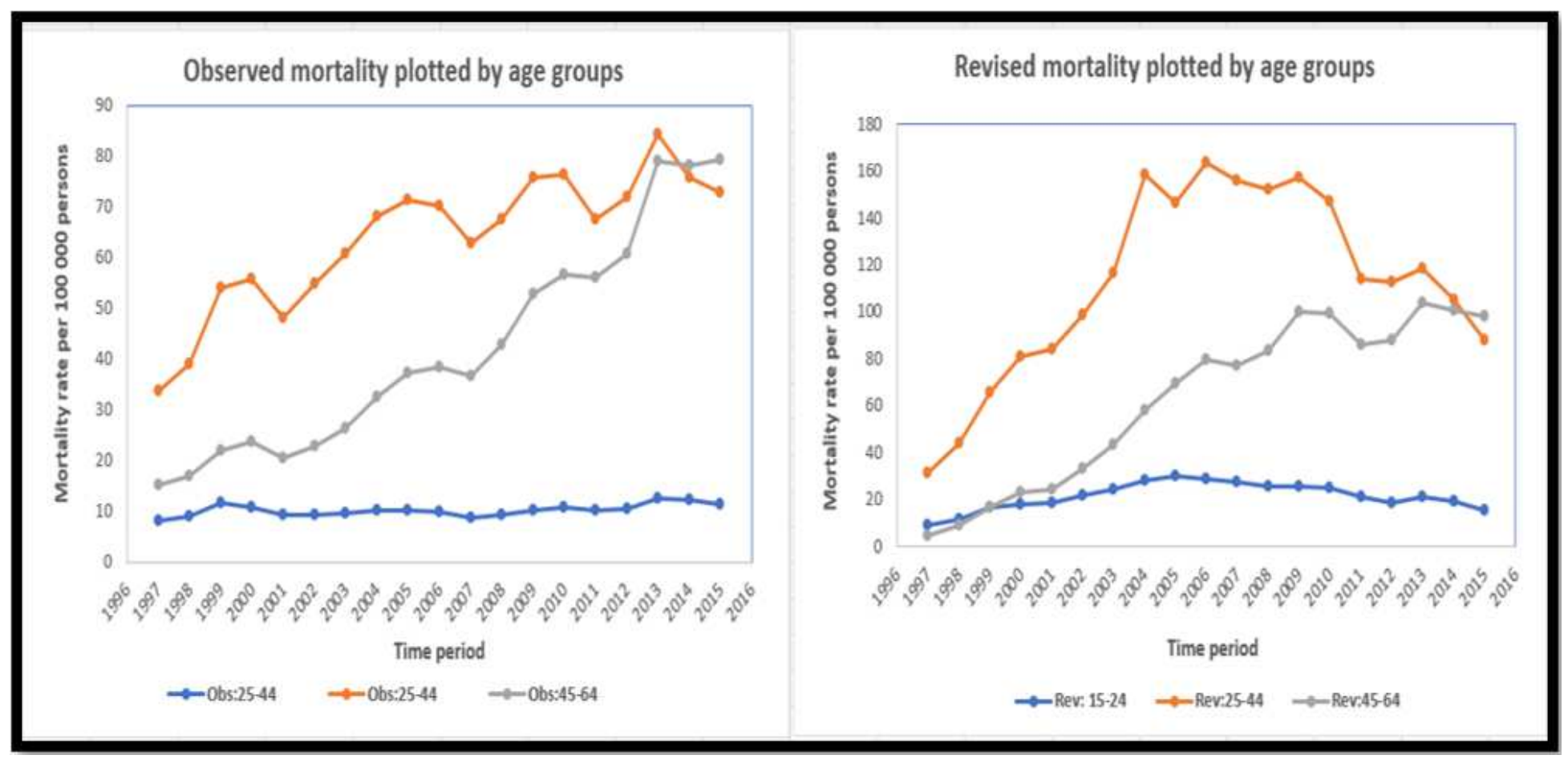

Figure 3

Observed and revised HIV-related mortality rates of the three age-groups

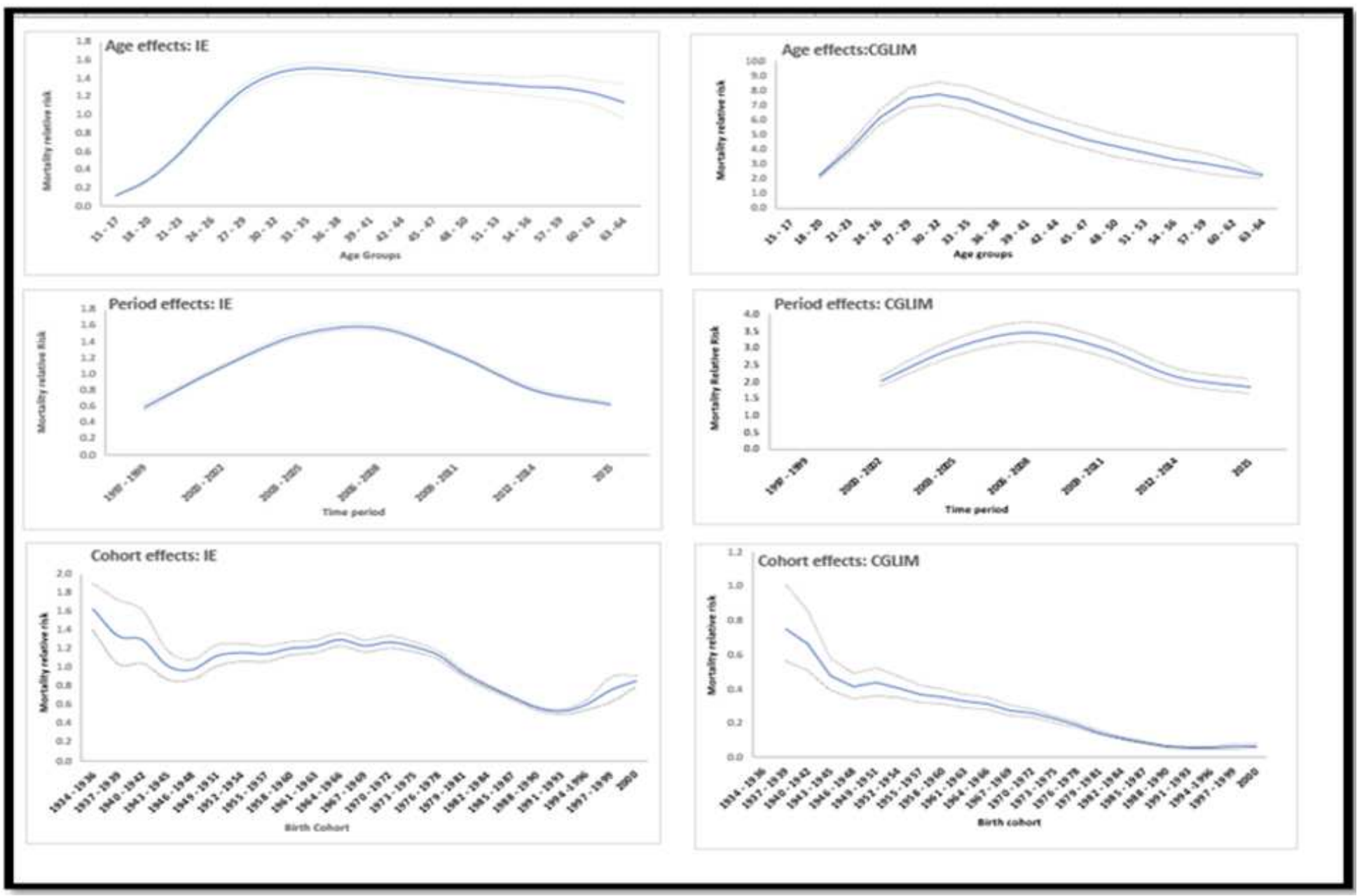


Figure 4

Age, period and cohort effects of TB mortality. Notes: dotted lines present $95 \%$ confidence intervals.

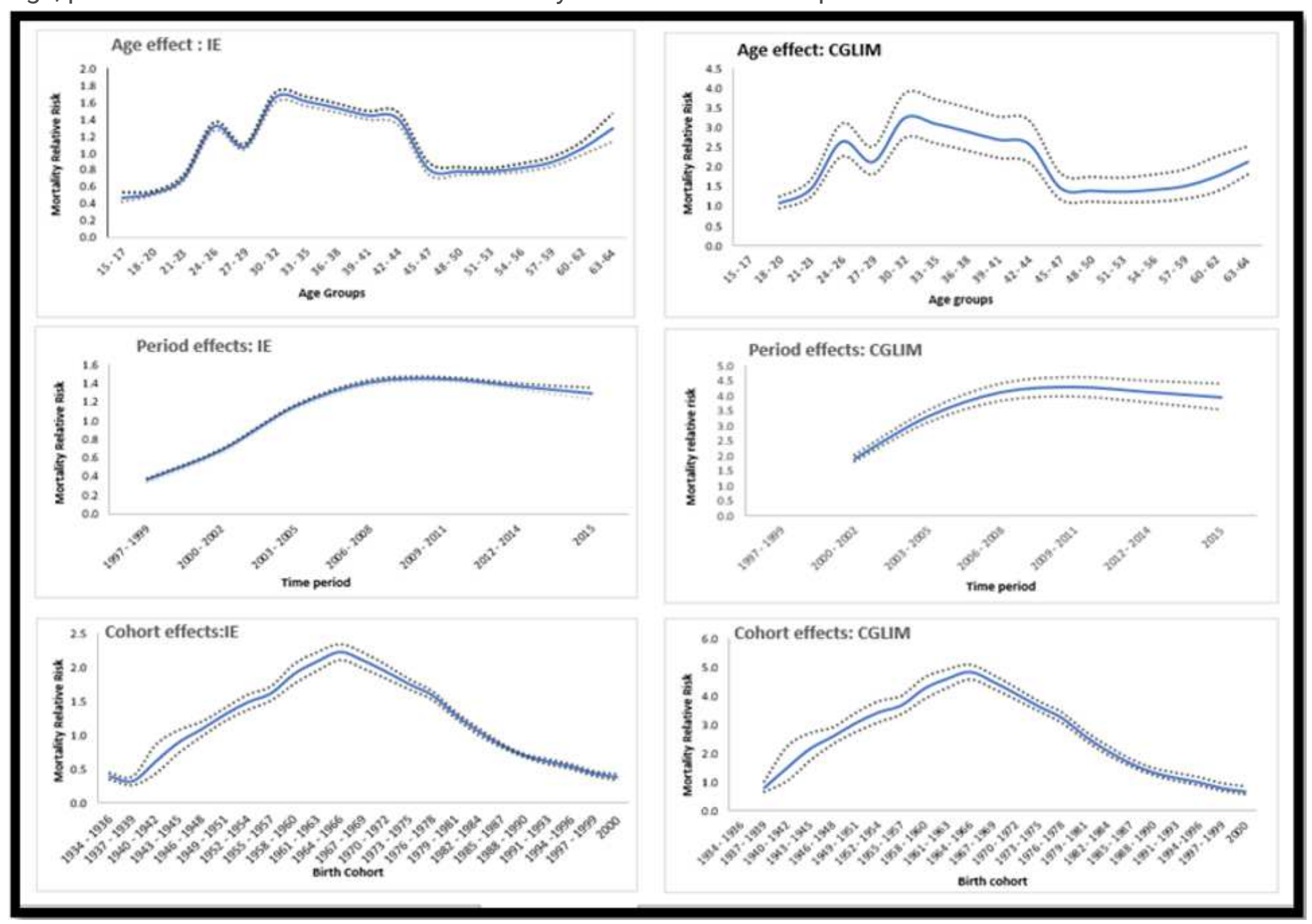

\section{Figure 5}

Age, period and cohort effects of HIV mortality. Notes: dotted lines present $95 \%$ confidence intervals. 